\title{
A Stochastic Approach to Design MIMO Antenna with Parasitic Elements Based on Propagation Characteristics
}

\author{
Naoki HONMA $^{\dagger a)}$, Member, Kentaro NISHIMORI ${ }^{\dagger \dagger}$, Senior Member, Riichi KUDO ${ }^{\dagger \dagger}$, Member, \\ Yasushi TAKATORI $^{\dagger \dagger \dagger}$, Senior Member, Takefumi HIRAGURI ${ }^{\dagger \dagger \dagger}$, and Masato MIZOGUCHI ${ }^{\dagger \dagger \dagger}$, Members
}

\begin{abstract}
SUMMARY This paper proposes a channel capacity maximization method for Multiple-Input Multiple-Output (MIMO) antennas with parasitic elements. Reactive terminations are connected to the parasitic elements, and the reactance values are determined to achieve stochastically high channel capacity for the environment targeted. This method treats the S-parameter and propagation channel of the antenna, including the parasitic elements, as a combined circuit. The idea of the 'parasitic channel,' which is observed at the parasitic antenna, is introduced to simplify the optimization procedure. This method can significantly reduce the number of necessary measurements of the channel for designing the antenna. As a design example, a bidirectional Yagi-Uda array, which has two driven antennas at both ends of the linear array, is measured in an indoor environment. The resulting design offers enhanced channel capacity mainly due to its improved signal-to-noise ratio compared to the antenna without the parasitic antennas.
\end{abstract}

key words: MIMO, parasitic antennas, mobile antennas, Yagi-Uda arrays

\section{Introduction}

The spatial multiplexing technique using multi-antennas known as Multiple-Input Multiple-Output (MIMO) continues to be pursued since it can enhance the spectral efficiency of wireless communications [1]. To implement MIMO in wireless communications systems, small antenna configurations suitable for compact terminals have been studied, such as PC card terminals, [2]-[4], PDAs (Personal Digital Assistants) [5], [6], and cellular phone terminals [7], [8].

However, if further channel capacity enhancement is required without increasing the aperture or the number of transmitters/receivers, the radiation pattern of the antennas must be considered. Jensen et al. [9] have shown that the MIMO-optimized aperture distribution can increase channel capacity. It was also shown that the non-conjugate matching condition improves the channel capacity since it gives radiation patterns with low correlation and fairly high signal-tonoise ratios (SNR) [10]. These studies indicate there is still room for optimizing the radiation pattern, even for the small MIMO antennas, and that the optimal radiation pattern de-

\footnotetext{
Manuscript received January 4, 2010.

Manuscript revised May 5, 2010.

${ }^{\dagger}$ The author is with Faculty of Engineering, Iwate University, Morioka-shi, 020-8551 Japan.

${ }^{\dagger}$ The author is with Niigata University, Niigata-shi, 950-2181

${ }^{\dagger \dagger}$ The authors are with NTT Network Innovation Laboratories, NTT Corporation, Yokosuka-shi, 239-0847 Japan.

${ }^{\dagger+\dagger \dagger}$ The author is with Faculty of Engineering, Nippon Institute of Technology, Saitama-ken, 345-8501 Japan.

a)E-mail: honma@m.ieice.org

DOI: $10.1587 /$ transcom.E93.B.2578
} Japan. pends on the propagation environment in which the terminal antennas are placed.

The use of parasitic antennas is the one of the easiest approaches for designing the radiation pattern.

The adaptive array with parasitic antennas have been well studied [11], [12], and the work [11] treats the antenna impedance and propagation channel as a combined circuit. However, it can be difficult to apply the adaptive array with the parasitic antennas for the compact terminal because of its limited size and battery power. Hence, such terminals need the antenna with the fixed radiation pattern, which gives high channel capacity even without adaptive radiation pattern control. Therefore, for small and simple terminals, designing fixed antenna based on stochastic approach is more suitable than the adaptive or reconfigurable array antenna. This paper proposes a practical design method for antennas with parasitic elements that yields stochastically high channel capacity. To find out optimal antenna parameter from enormous number of possible antennas, the propagation channel and the antenna characteristics, including those of the parasitic antennas, are treated as a combined circuit. The channel capacity is maximized by changing the electric lengths of the parasitic antennas virtually; this reduces the number of measurements since only the basic antenna configuration needs to be measured. In [13], the Yagi-Uda array is optimized using reactive termination, but it only focused the gain of the Yagi-Uda array. In order to find out fixed radiation pattern that maximize the channel capacity, the channel information and antenna characteristics must be combined. As an example, we optimize the design of a bidirectional Yagi-Uda array [14]. The bidirectional Yagi-Uda array is a variant of Yagi-Uda array, and conventionally it is designed for obtaining a high antenna gain with a small form factors. This study optimizes a bidirectional Yagi-Uda array to yield high channel capacity in the indoor environment.

In Sect. 2, the multi-antenna system model and proposed optimization method are described. In Sect. 3, the proposed design method is experimentally verified using the bidirectional Yagi-Uda array.

\section{Model and Proposed Design Method}

2.1 Multi-Antenna System Model Including Parasitic Antennas

Figure 1 shows the system model considered here. This 


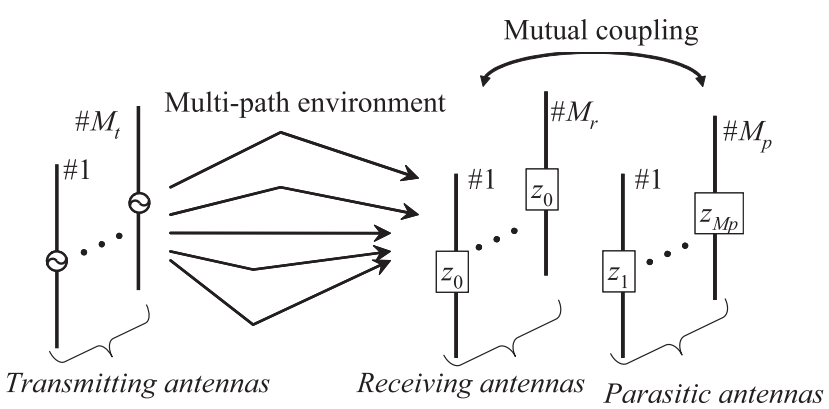

Fig. 1 Model of the multi-antenna system including the parasitic antennas.

model consists of $M_{t}$ transmitting antennas, $M_{r}$ receiving antennas, and $M_{p}$ parasitic antennas. The electric lengths of the parasitic antennas are virtually changed by using reactive terminations, $\left\{z_{1} \ldots z_{M p}\right\}$, at the parasitic antennas for simplicity. The receiving elements are connected to the loads, $z_{0}$, of the receiver through a matching and decoupling network [15] whose scattering parameter ( $\mathrm{S}$-parameter) is represented by $S_{M}$. In the following consideration and measurements, the value of the receiving port loads, $z_{0}$, is $50 \Omega$. Here, we define the S-parameter matrix, $S_{S}$, which is defined for all ports being terminated by the reference impedance $z_{0}$ and is partitioned as,

$$
\boldsymbol{S}_{S}=\left(\begin{array}{lll}
\boldsymbol{S}_{T T} & \boldsymbol{S}_{T R} & \boldsymbol{S}_{T P} \\
\boldsymbol{S}_{R T} & \boldsymbol{S}_{R R} & \boldsymbol{S}_{R P} \\
\boldsymbol{S}_{P T} & \boldsymbol{S}_{P R} & \boldsymbol{S}_{P P}
\end{array}\right) .
$$

where the subscripts $T, R$, and $P$ indicate the transmitting, receiving, and parasitic antennas, respectively. Partitioned matrices $\boldsymbol{S}_{T T}$ and $\boldsymbol{S}_{R R}$ are the S-parameters for the transmitting and receiving antennas, respectively. Term $S_{P P}$ is the $S$-parameter matrix of the parasitic antenna and $S_{P R}$ is the matrix that represents the mutual coupling between the receiving antenna and parasitic antenna. Terms $\boldsymbol{S}_{R T}$ and $\boldsymbol{S}_{P T}$ denote the channel matrices from the transmitting antenna to the receiving antenna and parasitic antenna, respectively. Since the S-parameter matrix is symmetric, $\boldsymbol{S}_{T R}=\boldsymbol{S}_{R T}^{T}$, $\boldsymbol{S}_{T P}=\boldsymbol{S}_{P T}^{T}$, and $\boldsymbol{S}_{R P}=\boldsymbol{S}_{P R}^{T}$.

Matrix $\boldsymbol{S}_{P T}$, which corresponds to the channel between the transmitting antenna and the parasitic antenna at the receiver, can not be measured in an actual situation, but it is possible to define $S_{P T}$ in this model, and it should be obtained by measurement. In this paper, we define $S_{P T}$ as the 'parasitic channel.'

The termination condition at the parasitic antenna is defined as

$$
\boldsymbol{\Gamma}=\left(\begin{array}{ccc}
\Gamma_{1} & & 0 \\
& \ddots & \\
0 & & \Gamma_{M p}
\end{array}\right),
$$

where $\Gamma_{i}$ is the reflection coefficient of the $\mathrm{i}$-th termination. It is defined as $\Gamma=\left(j z_{i}-z_{0}\right) /\left(j z_{i}+z_{0}\right)$, where $z_{i}$ can be expressed as $z_{i}=r_{i}+j x_{i}$. Figure 2 diagrams the S-parameter model for this multi-antenna system. Here, the parasitic antenna ports are terminated by the reactive loads, $\left\{z_{1} \ldots z_{M p}\right\}$.

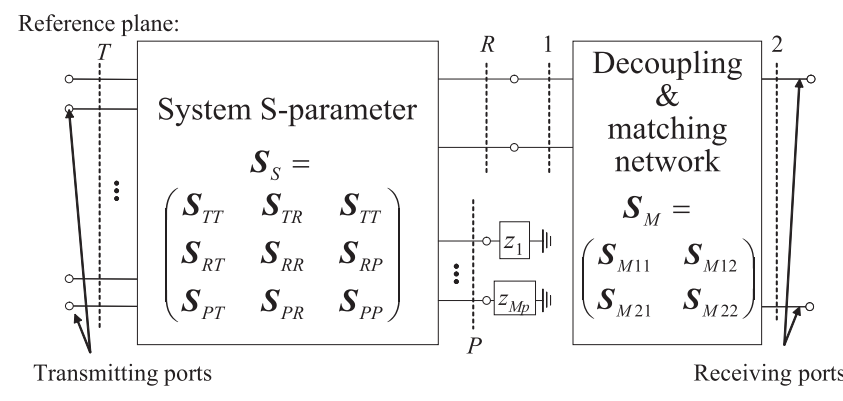

Fig. 2 Model of the multi-antenna system including parasitic antennas.

At the receiving antenna port, the decoupling and matching network (DMN) is connected, shown as $\boldsymbol{S}_{M}$ to consider the effect of the radiation pattern without the matching condition; it is described in the later part of this paper.

\subsection{Optimization of Parasitic Antenna}

The key idea of this paper is that the radiation pattern of the array antenna can be designed by appropriately setting the electric lengths of the parasitic antennas. Changing the electric length of the antenna is achieved by changing reactive load. This approach reduces the number of measurements needed since antennas with parasitic antennas of various lengths are not necessary.

Connecting a reactive termination to a parasitic antenna transforms the system's S-parameter as follows

$$
\begin{aligned}
& \boldsymbol{S}_{S}^{\prime}=\left(\begin{array}{ll}
\boldsymbol{S}_{T T}^{\prime} & \boldsymbol{S}_{T R}^{\prime} \\
\boldsymbol{S}_{R T}^{\prime} & \boldsymbol{S}_{R R}^{\prime}
\end{array}\right) \\
& =\left(\begin{array}{l}
\boldsymbol{S}_{T T}+\boldsymbol{S}_{P T}^{T}\left[\boldsymbol{\Gamma}^{-1}-\boldsymbol{S}_{P P}\right]^{-1} \boldsymbol{S}_{P T} \\
\boldsymbol{S}_{R T}+\boldsymbol{S}_{P R}^{T}\left[\boldsymbol{\Gamma}^{-1}-\boldsymbol{S}_{P P}\right]^{-1} \boldsymbol{S}_{P T}
\end{array}\right. \\
& \left.\begin{array}{c}
\boldsymbol{S}_{T R}+\boldsymbol{S}_{P T}^{T}\left[\boldsymbol{\Gamma}^{-1}-\boldsymbol{S}_{P P}\right]^{-1} \boldsymbol{S}_{P R} \\
\boldsymbol{S}_{R R}+\boldsymbol{S}_{P R}^{T}\left[\boldsymbol{\Gamma}^{-1}-\boldsymbol{S}_{P P}\right]^{-1} \boldsymbol{S}_{P R}
\end{array}\right),
\end{aligned}
$$

where the partitioned matrix, $S_{R T}^{\prime}$, is the channel matrix when the parasitic antennas are connected to the reactive terminations. This includes the effect of miss-matching. Since we are interested in the radiation pattern, not impedance matching, the decoupling and matching network (DMN) is introduced to verify the channel matrix properties under ideal matching conditions. The S-parameter matrix of the lossless DMN is easily obtained from reference [15], and is expressed as

$$
\boldsymbol{S}_{M}=\left(\begin{array}{ll}
\boldsymbol{S}_{M 11} & \boldsymbol{S}_{M 12} \\
\boldsymbol{S}_{M 21} & \boldsymbol{S}_{M 22}
\end{array}\right) .
$$

By connecting two circuits, $S_{S}^{\prime}$ and $S_{M}$, the observed channel matrix, $\boldsymbol{H}$, can be written as,

$$
\begin{aligned}
\boldsymbol{H}= & \boldsymbol{S}_{M 21} \cdot\left(\boldsymbol{I}-\boldsymbol{S}_{R R}^{\prime} \boldsymbol{S}_{M 11}\right)^{-1} \cdot \boldsymbol{S}_{R T}^{\prime} \\
= & \boldsymbol{S}_{M 21} \\
& \cdot\left[\boldsymbol{I}-\left\{\boldsymbol{S}_{R R}+\boldsymbol{S}_{P R}^{T}\left(\boldsymbol{\Gamma}^{-1}-\boldsymbol{S}_{P P}\right)^{-1} \boldsymbol{S}_{P R}\right\} \boldsymbol{S}_{M 11}\right]^{-1} \\
& \cdot\left[\boldsymbol{S}_{R T}+\boldsymbol{S}_{P R}^{T}\left(\boldsymbol{\Gamma}^{-1}-\boldsymbol{S}_{P P}\right)^{-1} \boldsymbol{S}_{P T}\right]
\end{aligned}
$$


Equation (5) shows that the channel matrix with an arbitrary termination condition can be easily estimated without further measurement if the 'parasitic channel,' $\boldsymbol{S}_{P T}$, is known. Here, the S-parameter of the receiver antennas, described as $S_{R R}, S_{P R}$, and $S_{P P}$, must also be known in advance.

The followings are the actual procedure for proposed optimization method. In the first step, the antenna configuration with the parasitic antennas must be determined, and this will determine the upper bound of the channel capacity. To obtain high channel capacity within the limited antenna aperture, the parasitic antenna must be coupled strongly, and placing the driven antennas around the edge of the aperture is desirable [9]. The $S$-parameter matrices, $\boldsymbol{S}_{R R}, \boldsymbol{S}_{P R}$, and $S_{P P}$, need to be measured. Here, the array antenna with the parasitic antennas is used at the receiver side in this consideration. In the second step, the transmitting and receiving antennas are placed in typical environments, where the array antenna is supposed to be used. The channel matrices with several locations and orientations of the array antenna are measured. Here, the parasitic channel, $\boldsymbol{S}_{P T}$, must be included in these measurements. In the third step, the termination condition is optimized so as to maximize the channel capacity, which can be calculated by

$$
C=\log _{2} \operatorname{det}\left(\boldsymbol{I}+\frac{\gamma}{M_{T}} \boldsymbol{H} \boldsymbol{H}^{H}\right)
$$

$\gamma$ is the SNR observed at the receiver. The channel matrix, $\boldsymbol{H}$, with arbitrary termination conditions can be calculated by using (5). Since the proposed method is for designing the MIMO antenna with stochastically high channel capacity, the termination impedances should be determined to maximize the outage capacity by giving various termination impedances, $\left\{z_{1} \ldots z_{M p}\right\}$ at the parasitic antennas. The advantage of the proposed method is that the channel with arbitrary terminations can be estimated without actually attaching them to the parasitic antennas. This mean the process of changing the terminations in the measurement can be omitted. The several optimization methods, such as Genetic Algorithm (GA) [22], Particle Swarm Optimization (PSO) [23], steepest gradient method, etc. have been proposed, but these issues are out of scope. Based on the procedure described above, the optimal value of $z_{i}$ is determined.

\section{Experimental Demonstration Using Bidirectional Yagi-Uda Array}

\subsection{Antenna Configuration}

As a demonstration, some results of parasitic antenna optimization are shown and discussed. Figure 3 shows the conceptual sketch of the bidirectional Yagi-Uda array antenna. A Yagi-Uda array is well-known; it uses parasitic antennas to form the radiation pattern. When two or more Yagi-Uda arrays are used for MIMO communication, the overall size of the antenna becomes too large to suit compact terminals. In the bidirectional Yagi-Uda array, two opposed Yagi-Uda arrays share director elements, and this configuration offers high gain characteristics even if there are driven antennas at both ends of the array. Note the parasitic antennas couple more strongly to the driven antennas when they are placed in the center of the array than when they are placed at the ends of the array [16]. In terms of aperture efficiency, placing the driven antennas at the end of the array is effective in obtaining high MIMO channel capacity [9]. In this consideration, the reactive terminations, $z_{1}=j x_{1}$, and $z_{2}=j x_{2}$ are given equally, i.e., $z_{1}=z_{2}=j x_{1}$, since the arrival direction of the signal can be random in the indoor situation and the antenna should have symmetrical radiation pattern.

Table 1 shows the antenna configurations measured here. The transmitting antenna is an 8-element circular array antenna, and the antenna spacing is set to a $\lambda_{0}$. In configuration (A), a 4-element dipole array is used as the receiving antenna. The central two antennas are assumed to be the parasitic antenna, nevertheless, the channel including the parasitic antennas must be measured, and the channel response with the reactive loads at the parasitic antennas are estimated as described in 2. For comparison, the antenna without the parasitic antennas, which is shown in Table 1 (B), is also evaluated.

\subsection{Measurement Setup}

The detail measurement conditions are listed in Table 2 . The frequency was $4.85 \mathrm{GHz}$, and the receiving antenna, a 4element dipole array, was placed at 9 measurement points, each separated by $3 \mathrm{~m}$; the channels created by setting the receiving antenna at 8 different horizontal orientations were measured at each point. Therefore, the total number of the channel sample is $53 \times 9 \times 8=3816$. The height of the transmitting antenna was set to $1.5 \mathrm{~m}$, slightly higher than

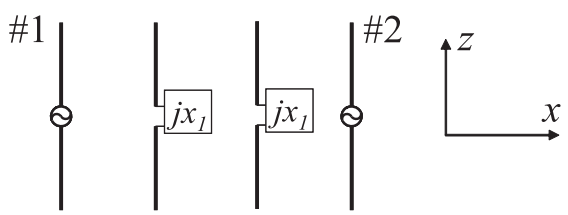

Fig. 3 Conceptual sketch of bidirectional Yagi-Uda array.

Table 1 Antenna combinations.

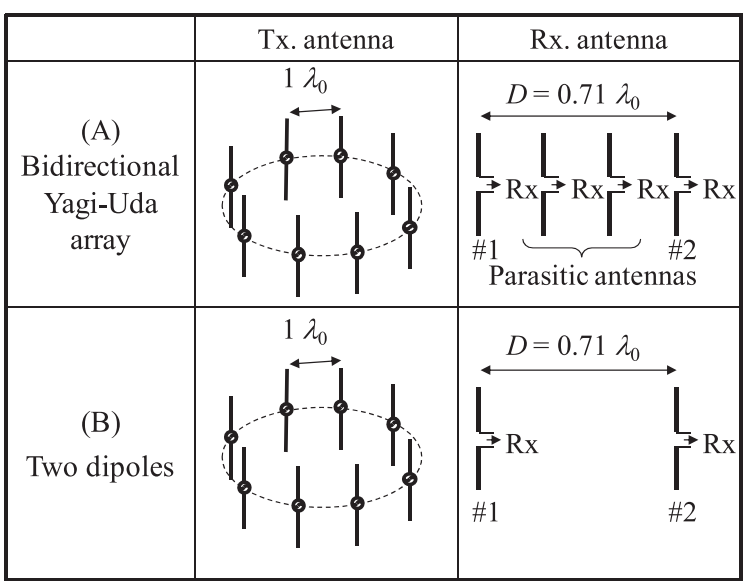


Table 2 Measurement conditions.

\begin{tabular}{|c||c|}
\hline Frequency & $4.85 \mathrm{GHz}$ \\
\hline Bandwidth & $20 \mathrm{MHz}$ \\
\hline Number of subcarriers & 53 \\
\hline Rx. antenna direction & $0 \sim 360$ deg. (45 deg. step) \\
\hline Rx. antenna positions & 9 (see Fig. 4 ) \\
\hline Rx. antenna height & $1.2 \mathrm{~m}$ \\
\hline Tx. antenna position & 1 (see also Fig. 4 ) \\
\hline Tx. antenna height & $1.5 \mathrm{~m}$ \\
\hline
\end{tabular}

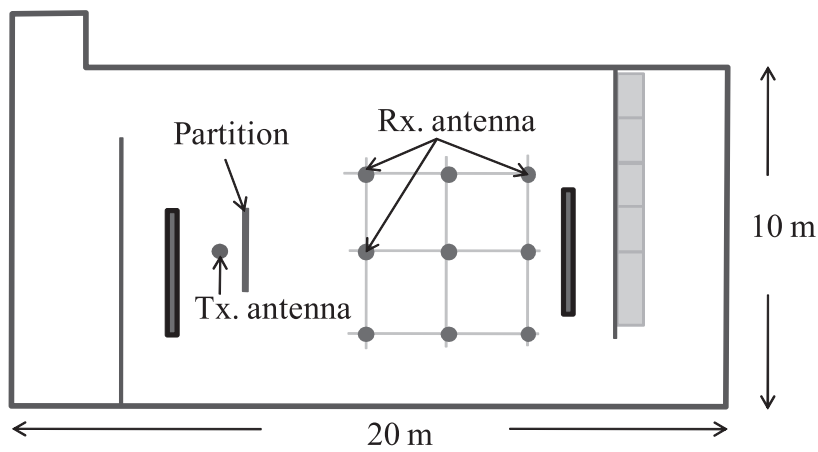

Fig. 4 Indoor environment.

the receiving antenna. The SNR in this study is evaluated by measuring the noise power before the signals are transmitted. Figure 4 is a sketch of the measurement environment. The height, width, and depth of the room were 4, 20 , and $10 \mathrm{~m}$, respectively. The transmitting antenna was placed at the left side of the room, and the receiving antenna was placed at the measurement points indicated in the figure. The NLOS situation was created by placing a partition board in front of the transmitting antenna. To prevent diffraction from the edge of the board, it was covered with an anechoic material.

A photo of the antennas and environment is shown in Fig. 5. The 4-element dipole array was placed on a fixture that could rotate the direction of antenna horizontally. Fig. 5(b) shows the LOS situation; the transmitting antenna is also shown on top of the pole. The testbed [19] can be seen behind the receiving array antenna; four receivers of the MIMO testbed were used in this measurement.

\subsection{Results}

Figure 6 plots median capacity versus reactance, $x_{1}$. This graph was generated from the results at the various positions and directions of receiving antenna. It is found that the channel capacity at the range of $x_{1}<-200 \Omega$ slightly varies, and the maximum channel capacity occurs around $x_{1}=-290 \Omega$ in both $\operatorname{LOS}(\mathrm{a})$ and NLOS(b) environments. The channel capacity improvement compared with two dipoles in the NLOS environment is greater than that in the LOS environment. It is also found that a drop in channel capacity is observed at $x_{1}=-47 \Omega$ in both $\operatorname{LOS}(\mathrm{a})$ and $\operatorname{NLOS}(\mathrm{b})$ environments. From these results, the values of $x_{1}$ that give maximum and minimum capacity are independent of the existence of the direct path in this environment. In order to find

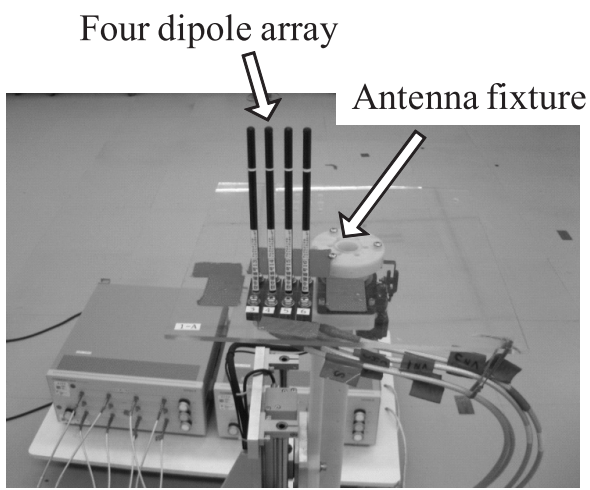

(a) Receiving array antenna

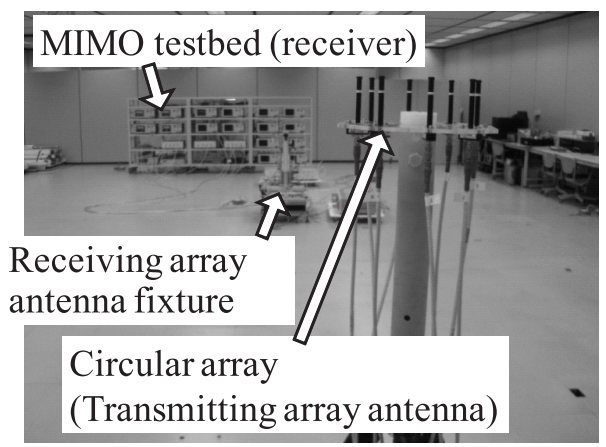

(b) Measurement setup

Fig. 5 Photo of antennas and measurement setup.
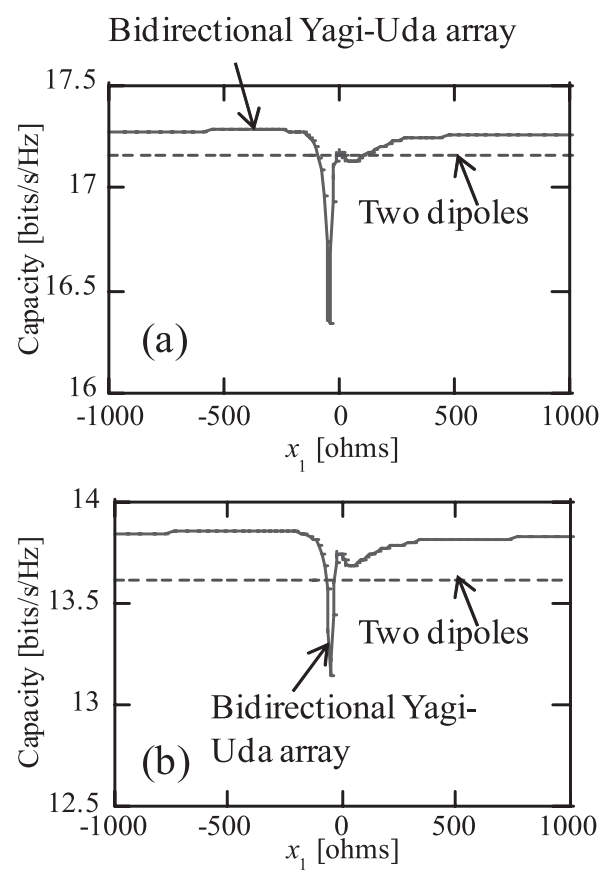

Fig. 6 Median capacity versus the reactance value, $x_{1} .:$ (a) LOS environment, (b) NLOS environment.

what is happening at these $x_{1}$ values, the SNR and the spatial correlation is investigated as in the following Figs. 7 and 8 .

Figure 7 plots SNR versus reactance. Similarly to the 

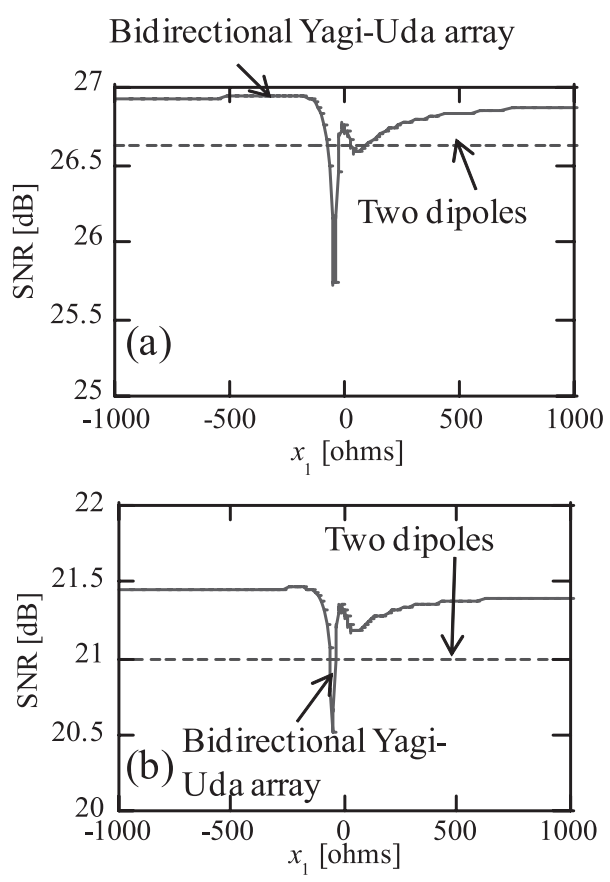

Fig. 7 Median SNR versus the reactance value, $x_{1} .:$ (a) LOS environment, (b) NLOS environment.
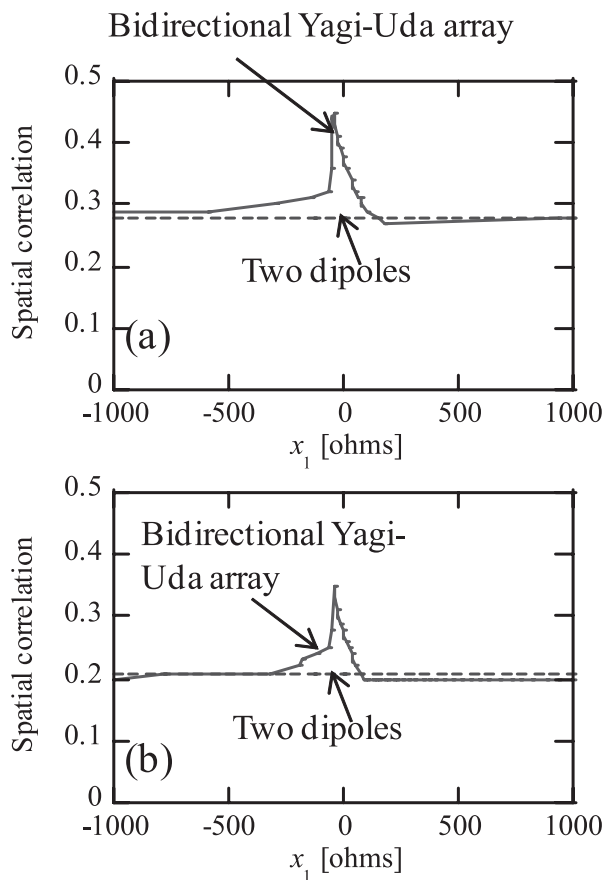

Fig. 8 Median spatial correlation versus the reactance value, $x_{1} .:$ (a) LOS environment, (b) NLOS environment.

capacity, the parasitic antennas offer better improvement in the NLOS environment than in the LOS environment.

Figure 8 shows the characteristics of the spatial correlation versus $x_{1}$. The spatial correlation rises when $x_{1}$ is around -47 , which corresponds to the minimum point in the channel capacity as shown in Fig. 6. The difference in the spatial correlation between the bidirectional Yagi-Uda array and two dipoles is very small. Here, the spatial correlation is
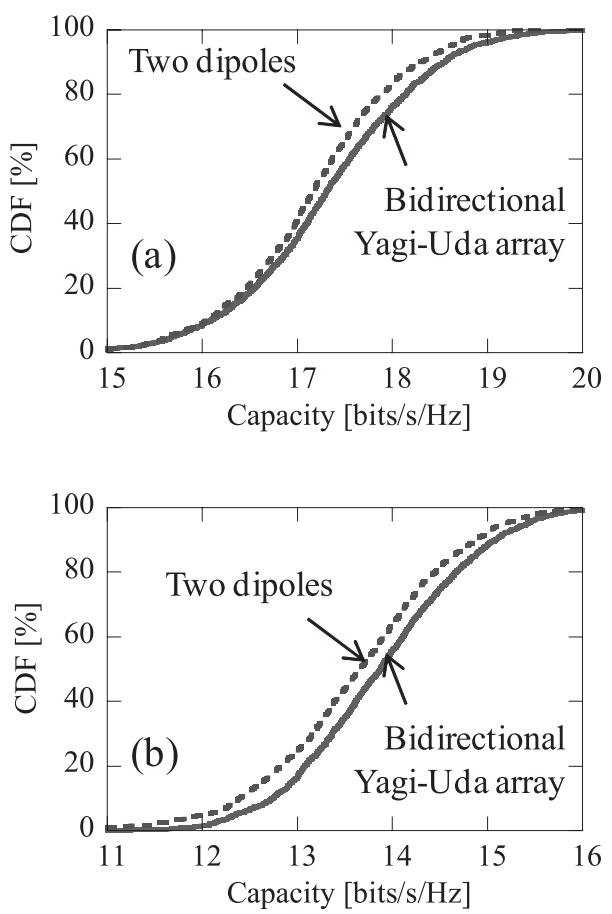

Fig.9 Cumulative distribution function of channel capacity with the MIMO-optimal condition $\left(x_{1}=-290 \Omega\right.$ ), (a) LOS environment, (b) NLOS environment.

larger than 0 even with DMN is used. This is because DMN only orthogonalizes the radiation patterns of the array antenna but does not orthogonalize the channel [20]. In [21], it is described the channel capacity is hardly affected by the spatial correlation when it is lower than 0.9. From the reason discussed here and the results of the measurement shown in Fig. 6 8, it is found that the parasitic antennas can improve the channel capacity by better collecting the power delivered to the aperture.

The cumulative distribution function (CDF) of the channel capacity is indicated in Fig. 9. $x_{1}$ is set to $-290 \Omega$, which maximizes the median channel capacity. It can be seen that the overall channel capacity is improved even when the optimization is performed only for median capacity. Also, the channel capacity is improved more in the NLOS environment than in the LOS environment.

Figure 10 shows the CDF of the SNR. The distribution of the SNR is very similar to that of the capacity. In the LOS environment the improvement is remarkable in higher percentage range, and this means that the SNR tends to be enhanced for the receiving antennas in not bad environment.

Figure 11 shows the $\mathrm{CDF}$ of the spatial correlation. In the LOS situation, the spatial correlation with optimal $x_{1}$ is higher than that achieved with two dipoles. The radiation pattern that provides the stochastically highest channel capacity enhances the capacity only by improving the SNR stochastically. On the other hand, the spatial correlation of the bidirectional Yagi-Uda array in the NLOS environment is very similar to that with the two dipoles. In the NLOS situation, the optimized pattern only slightly impacts the spatial correlation. 

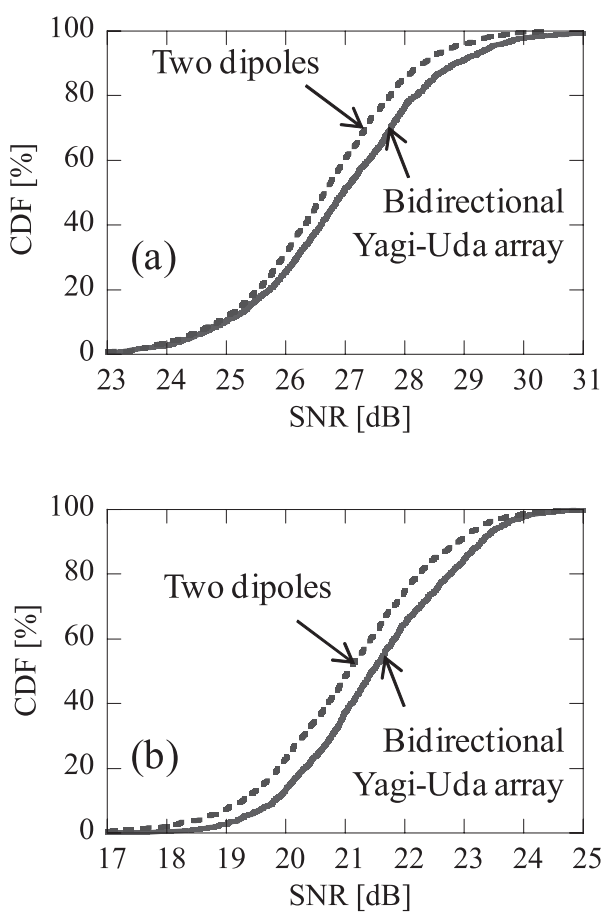

Fig. 10 Cumulative distribution function of SNR with the MIMOoptimal condition $\left(x_{1}=-290 \Omega\right.$ ), (a) LOS environment, (b) NLOS environment.
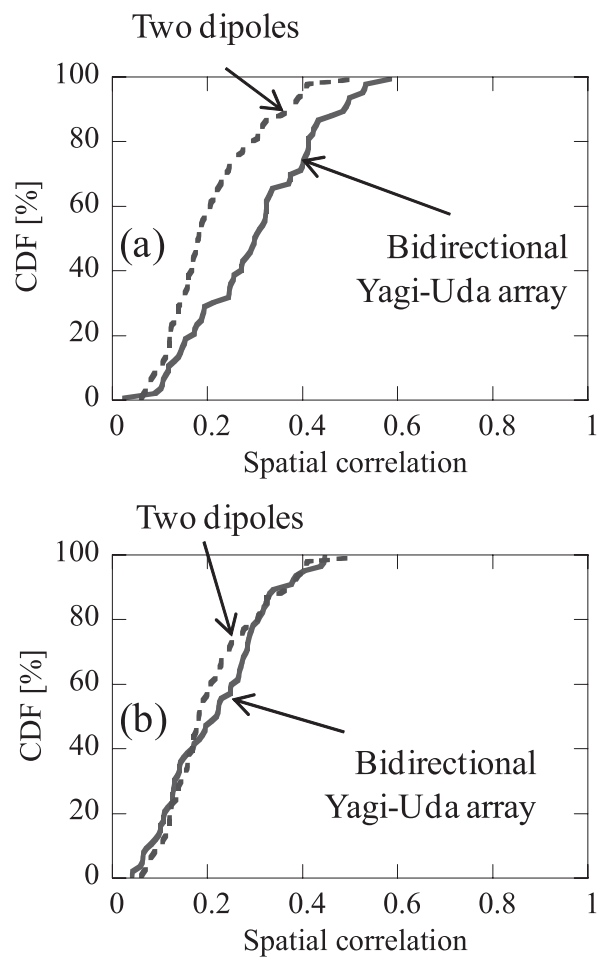

Fig. 11 Cumulative distribution function of spatial correlation with the MIMO-optimal condition ( $x_{1}=-290 \Omega$ ), (a) LOS environment, (b) NLOS environment.

Figure 12 plots antenna gain versus $x_{1}$. The antenna gains at the forward direction $(+x)$ and backward direction $(-x)$ are indicated. The radiation characteristics were obtained by exciting antenna \#1; antenna \#2 was terminated
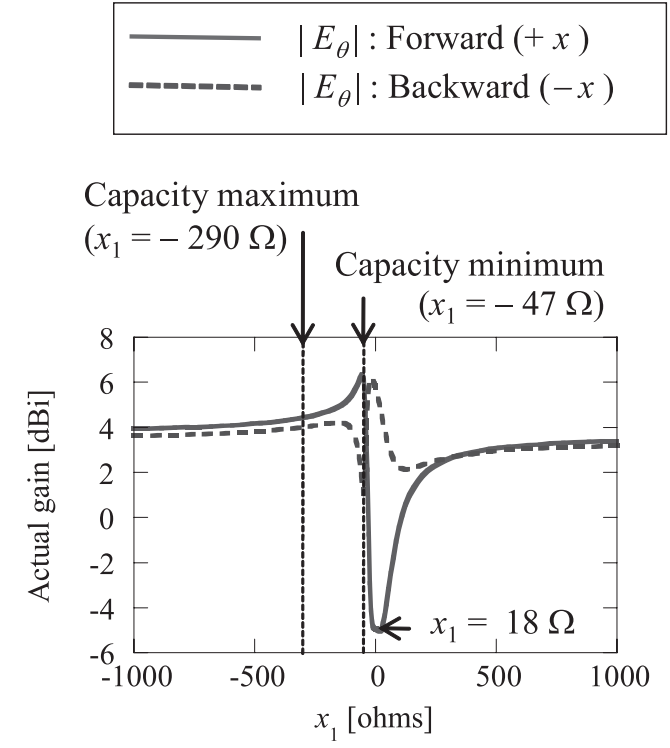

Fig. 12 Radiation pattern characteristics versus reactance value, $x_{1}$.

using a $50 \Omega$ resistor. It is found that the direction of the beam changes greatly around $-50 \leq x_{1} \leq 50$. The highest antenna gain at forward direction $(+x)$ is observed when $x_{1}=-47 \Omega$. This can be recognized as a director mode since the beam extends away from the driven antenna in the direction of the parasitic antennas. In [13], the optimization is performed for this gain maximum, and this mode has been used conventionally to enhance the antenna gain, however it corresponds to the minimum capacity point shown in Fig. 6 . This means the optimal design for MIMO communication differs from that of the conventional Yagi-Uda array. In the region of $x_{1}>0$, the parasitic antennas work as reflectors since the gain at $-x$ is higher than that at $+x$. In this mode, the spatial correlation is slightly lower than in the director mode, see Fig. 8, however, the low SNR affects the channel capacity.

Figure 13 show the measured radiation patterns; the specific termination conditions are shown. The patterns in the horizontal plane were measured ( $x y$-plane) when antenna \# 1 was excited. The radiation pattern at the capacity maximum is almost symmetrical with respect to $y z$-plane, that is, the radiation patterns of \#1 and \#2 are very similar. It will cause a relatively high spatial correlation in the observed channel. However, the increase in the spatial correlation is very slight, and the radiation pattern formed offers high SNR. Since the most of the arriving paths distribute in horizontal direction [18], the improvement in SNR is caused by the average gain enhancement in the horizontal plane, which is provided by the parasitic antennas. The director mode, which offers the highest antenna gain but the lowest capacity, forms the mainlobe in the direction of $\phi=0(+x)$. However, a high backlobe is seen at $\phi=180^{\circ}(-x)$ because of the other driven antenna at the end of the array. The condition, $x_{1}=18 \Omega$, gives the reflector mode, and the highest gain is observed at $\phi=180^{\circ}(-x)$. The maximum ratio of the gain at $\phi=180^{\circ}$ to that at $\phi=0$ is obtained in this mode, 


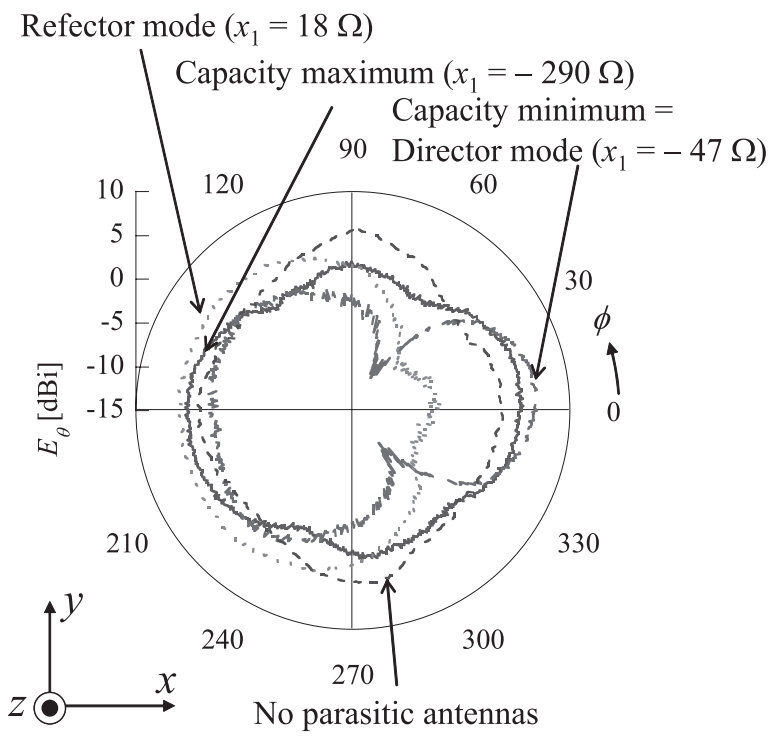

Fig. 13 Measured radiation pattern.

however, this cannot reduce the spatial correlation as shown in Fig. 8. This means that the simple combination of orthogonal radiation patterns cannot enhance the channel capacity, and the propagation characteristics should be taken into account in designing MIMO-optimal antennas.

The discussion above validates the proposed design method for the MIMO antennas using fixed parasitic antennas, and the demonstrated design achieved the highest median capacity at both LOS and NLOS situation in this environment even with the common reactance value. To obtain the universally optimal design for most indoor or other environments, more measurements at various situations can be needed. By this process, the antenna design parameter for typical environments can be extracted by proposed method. This is our future work.

\section{Conclusion}

A practical design method for antennas with the parasitic elements, which gives stochastically high channel capacity, has been proposed. In this method, the antenna characteristics and the propagation channel, which includes the parasitic antennas, are treated as a combined circuit, and the termination conditions of the parasitic antennas are optimized. A bidirectional Yagi-Uda array has been optimized and measured based on real channel distributions. The results of the measurements have indicated that the antenna designed by the proposed method can offer enhanced channel capacity mainly by its improved the signal-to-noise ratios. It was also found the mode for the maximum channel capacity differs from the gain maximum mode for the conventional YagiUda array, that is, MIMO-optimal design is needed. These results supports that a high capacity MIMO antenna with parasitic antennas can be designed with very small numbers of the channel measurement.

\section{Acknowledgements}

The authors thank Mr. Masaaki Ida and Mr. Yoshinobu Makise of NTT Advanced Technology Corporation for offering a great help in the measurement.

\section{References}

[1] I.E. Teletar, "Capacity of multi-antenna Gaussian channels," Tech. Rep., AT\&T-Bell Labs, June 1995.

[2] D.W. Browne, M. Mateghi, M.P. Fitz, and Y. Rahmat-Samii, "Experiments with compact antenna arrays for MIMO radio communications," IEEE Trans. Antennas Propag., vol.54, no.11, pp.32393250, Nov. 2006

[3] Y. Okano and K. Cho, "Monopole antenna array arrangement for card-type mobile terminal," 2004 IEEE Radio and Wireless Conference, pp.415-418, Sept. 2004.

[4] N. Honma, K. Nishimori, T. Seki, K. Nishikawa, and K. Tsunekawa, "Triple polarization antenna employing capacitor loaded monopole antenna and notch antenna for MIMO systems," 2005 International Symposium on Antennas and Propagation (ISAP 2005), vol.2, TB24, pp.367-370, Aug. 2005.

[5] C.C. Chiau, X. Chen, and C.G. Parini, "A compact four-element diversity-antenna array for PDA terminals in a MIMO system," Microwave and Optical Technology Letters, vol.44, no.5, pp.408-412, Jan. 2005.

[6] L. Schulteis, C. Kuhnert, and W. Wiesbeck, "Three dual-band miniaturized inverted $\mathrm{F}$ antennas integrated in a PDA for MIMO applications," 2006 IEEE Antennas and Propagation Society International Symposium, pp.3593-3596, July 2006.

[7] P. Hongli, L. Qizhong, and S. Tang, "The implementation of compact multiple antennas for WCDMA MIMO handset," 2003 IEEE Antennas and Propagation Society International Symposium, vol.1, pp.479-482, June 2003.

[8] Y. Gao, C.C. Chiau, X. Chen, and C.G. Parini, "Modified PIFA and its array for MIMO terminals," IEE Proc. Microw., Antennas Propag., vol.152, no.4, pp.255-259, Aug. 2005.

[9] M.A. Jensen, B. Quist, and N. Bikhazi, "Antenna design for mobile MIMO systems," IEICE Trans. Commun., vol.E91-B, no.6, pp.1705-1712, June 2008.

[10] B.K. Lau, J.B. Andersen, G. Kristensson, and A.F. Molisch, "Impact of matching network on bandwidth of compact antenna arrays," IEEE Trans. Antennas Propag., vol.54, no.11, pp.3225-3238, Nov. 2006

[11] D. Pinchera, J.W. Wallace, M.D. Migliore, and M.A. Jensen, "Experimental analysis of a wideband adaptive-MIMO antenna," IEEE Trans. Antennas Propag., vol.56, no.3, pp.908-913, March 2008.

[12] T. Ohira, and K. Gyoda,"Electronically steerable passive array radiator antennas for low-cost analog adaptive beamforming," 2000 IEEE International Conference on Phased Array Systems and Tech., pp.101-104, May 2000.

[13] J.H. Bojsen, H. Schjaer-Jacobsen, E. Nilsson, and J. Bach Anderson,"Maximum gain of Yagi-Uda arrays," Electron. Lett., vol.7, no.18, pp.531-532, Sept. 1971

[14] N. Honma, T. Seki, T.K. Nishikawa, K. Tsunekawa, and K. Sawaya, "Compact six-sector antenna employing three intersecting bidirectional microstrip Yagi-Uda arrays with common director," IEEE Trans. Antennas Propag., vol.54, no.11, pp.3055-3062, Nov. 2006.

[15] J. Weber, C. Volmer, K. Blau, R. Stephan, and M.A. Hein, "Miniaturized antenna arrays using decoupling networks with realistic elements," IEEE Trans. Microw. Theory Tech., vol.54, no.6, pp.27332740, June 2006.

[16] M.D. Migliore, D. Pinchera, and F. Schettino, "Improving channel capacity using adaptive MIMO antennas," IEEE Trans. Antennas Propag., vol.54, no.11, pp.3481-3489, Nov. 2006. 
[17] A.F. Molisch, "A generic model for MIMO wireless propagation channels in macro- and microcells," IEEE Trans. Signal Process., vol.52, no.1, pp.61-71, Jan. 2004.

[18] K. Nishimori, N. Tachikawa, Y. Takatori, R. Kudo, and K. Tsunekawa, "Frequency correlation characteristics due to antenna configurations in broadband MIMO transmission," IEICE Trans. Commun., vol.E88-B, no.6, pp.2438-2445, June 2005.

[19] K. Nishimori, R. Kudo, N. Honma, Y. Takatori, A. Ohta, and K. Okada, "Experimental evaluation using $16 \times 16$ multiuser MIMO testbed in an actual indoor scenario," 2008 IEEE Antennas and Propagation Society International Symposium, vol.2, no.204.2, July 2008.

[20] J.W. Wallace and M.A. Jensen, "Mutual coupling in MIMO wireless systems: A rigorous network theory analysis," IEEE Trans. Wirel. Commun., vol.3, no 4, pp.1317-1325, July 2004.

[21] S. Loyka and J. Mosig, "Channel capacity of N-antenna BLAST architecture," Electron. Lett., vol.36, no.7, pp.660-661, March 2000.

[22] R.L. Haupt, "An introduction to genetic algorithms for electromagnetics," IEEE Antennas Propag. Mag., vol.37, no.2, pp.7-15, April 1995.

[23] J. Robinson and Y. Rahmat-Samii, "Particle swarm optimization in electromagnetics," IEEE Trans. Antennas Propag., vol.52, no.2, pp.397-407, Feb. 2004.

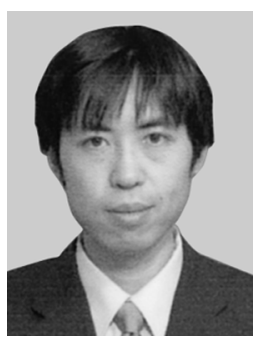

Naoki Honma received the B.E., M.E., and $\mathrm{Ph} . \mathrm{D}$. degrees in electrical engineering from Tohoku University, Sendai, Japan in 1996, 1998, and 2005, respectively. In 1998, he joined the NTT Radio Communication Systems Laboratories, Nippon Telegraph and Telephone Corporation (NTT), in Japan. He is now working for Iwate University. He received the Young Engineers Award from the IEICE of Japan in 2003, the APMC Best Paper Award in 2003, and the Best Paper Award of IEICE Communication Society in 2006, respectively. His current research interest is planar antennas for high-speed wireless communication systems. He is a member of IEEE.

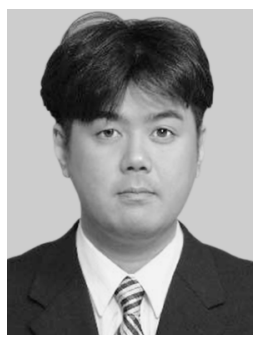

Kentaro Nishimori received the B.E., M.E. and $\mathrm{Ph} . \mathrm{D}$. degrees in electrical and computer engineering form Nagoya Institute of Technology, Nagoya, Japan in 1994, 1996 and 2002, respectively. In 1996, he joined the NTT Wireless Systems Laboratories, Nippon Telegraph and Telephone Corporation (NTT), in Japan. He was senior research engineer on NTT Network Innovation Laboratories. He is now associate professor in Niigata University. He was a visiting researcher at the Center for Teleinfrastructure (CTIF), Aalborg University, Aalborg, Denmark from Feb. 2006 to Jan. 2007. He was an editor for the Transactions on Communications for the IEICE Communications Society from May 2007 to May 2010 and Assistant Secretary of Technical Committee on Antennas and Propagation of IEICE from June 2009 to May 2010. He received the Young Engineers Award from the IEICE of Japan in 2001, Young Engineer Award from IEEE APS Japan Chapter in 2001, Best Paper Award of Software Radio Society in 2007 and Distinguished Service Award from the IEICE Communications Society in 2005 and 2008. His main interests are spatial signal processing including MIMO systems and interference management techniques on heterogeneous networks. He is a member of IEEE.

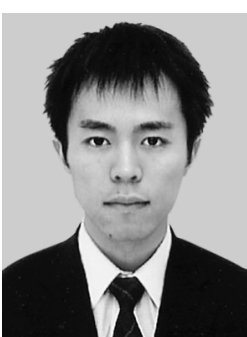

Riichi Kudo received the B.S. and M.S. degree in geophysics from Tohoku University, Japan, in 2001 and 2003, respectively. In 2003, he joined NTT Network Innovation Laboratories, Yokosuka, Japan. He has been engaged in the MIMO communication systems and the beamforming method. He received the Young Engineer Award from the Institute of Electronics, Information and Communication Engineers (IEICE) Japan in 2006. His current research interest is digital signal processing for wireless and optical communication systems. He is a member of IEEE.

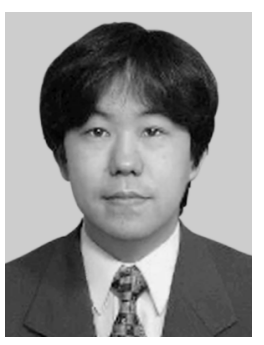

Yasushi Takatori was born in Tokyo, Japan, 1971. He received his B.E. degree in electrical and communication engineering and his M.E. degree in system information engineering from Tohoku University, Sendai, Japan in 1993 and 1995, respectively. He received his Ph.D. degree in wireless communication engineering from Aalborg University, Denmark in 2005. In 1995, he joined the Wireless Systems Laboratories, Nippon Telegraph and Telephone Corporation (NTT), in Japan. He is currently a senior research engineer in the Wireless Systems Innovation Laboratory of NTT Network Innovation Laboratories. He was a visiting researcher at the Center for TeleInFrastructure (CTIF), Aalborg University, Aalborg, Denmark from 2004 to 2005. He received the Young Engineers Award from the IEICE of Japan in 2000, the Excellent Paper Award of WPMC in 2004 and YRP Award in 2005. His current research interest is MIMO systems, spatial signal processing techniques and ultra-high speed optical communication systems. He is a member of the IEEE.

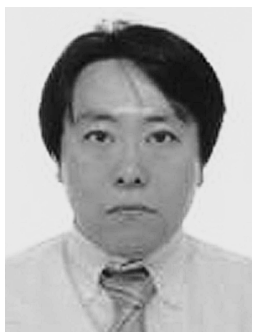

Takefumi Hiraguri received the M.E. and Ph.D. degrees from the University of Tsukuba, Ibaraki, Japan, in 1999 and 2008, respectively. In 1999, he joined the NTT Access Network Service Systems Laboratories, Nippon Telegraph and Telephone Corporation (NTT) in Japan. He has been engaged in research and development of MAC protocol for the high speed and the high communication quality in wireless LANs systems. He is now associate professor in Nippon Institute of Technology (NIT). He is a

member of IEEE.

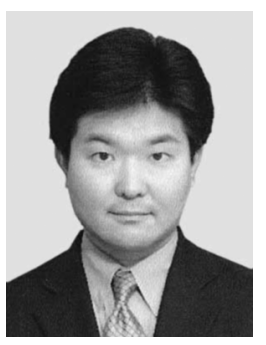

Masato Mizoguchi received the B.E. and M.E. degrees in electrical engineering from Tokyo University of Science, Japan in 1989 and 1991, respectively. In 1991, he joined Nippon Telegraph and Telephone Corporation (NTT) and was mainly engaged in research and development of personal communication systems and high data rate wireless LANs including the IEEE 802.11a systems. He is currently a Senior Research Engineer, Supervisor in the department of Wireless Systems Innovation Laboratory at NTT Network Innovation Laboratories, and is working on research and development of next generation wireless home network. He received the Young Researcher's Award in 1998, the Best Paper Award in 2000 and the Achievement Award in 2006 from IEICE. He is a member of IEEE. 